\title{
FOUR-DIMENSIONAL CHARACTERIZATION OF PAPER WEB AT THE WET END
}

\author{
Final Report for
}

American Forest \& Paper Association

and

Department of Energy's Office of Industrial Technologies

Principal Investigator:

James S. Goddard, Jr.

Image Science and Machine Vision Group

Oak Ridge National Laboratory*

Bethel Valley Road

Oak Ridge, TN 37831-6011

Phone: (865) 574-9034 Fax: (865) 576-8380

email: goddardjsjr@ornl.gov 


\begin{abstract}
This report presents a detailed description of a vision system whose purpose is to detect and to localize the nonuniformities that appear on the paper slurry (wood fiber and water mixture) at the wet end of a paper machine. Specifically, the system is capable of monitoring the paper slurry upon its exit from the headbox and alerting the operators of any event (e.g., streaks) that disrupts the otherwise homogeneous background. Such events are thought to affect crucial product properties such as formation, which if poor, results in thick and thin spots on the sheet and impacts its strength and printability. This report describes the vision system in terms of its hardware modules, as well as the image processing algorithms that it utilizes to perform its function. Basically, the system acquires both intensity and topographic information from the scene and uses texture-based features for the detection, and facet-based descriptors for the localization of the nonuniformities. In addition to being tested in a laboratory environment, a prototype of this system was constructed and deployed to a paper mill, where its performance was evaluated under realistic conditions. Installed on a fourdrinier paper machine, running at $480 \mathrm{~m} / \mathrm{min}$ and producing linerboard material, the vision system was able to monitor an approximately 1-meter-wide area and to successfully detect and localize slurry streaks.
\end{abstract}




\section{Table of Contents}

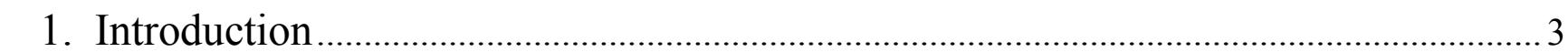

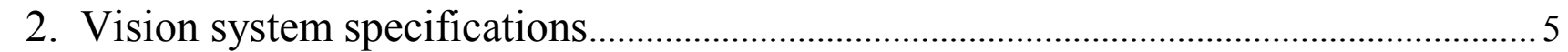

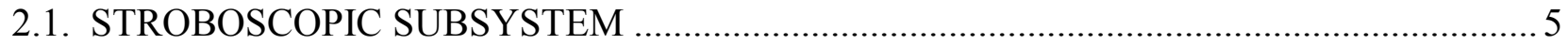

2.2. SURFACE-PROFILING SUBSYSTEM ………..........................................................

3. Image analysis for streak detection and localization ................................................ 9

3.1. TEXTURE-BASED STREAK DETECTION ALGORITHM ……................................. 10

3.2. FACET-BASED STREAK LOCALIZATION ALGORITHM...................................... 14

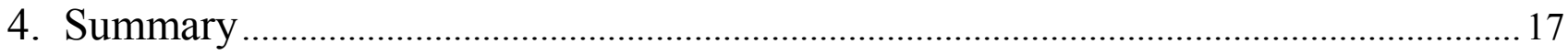

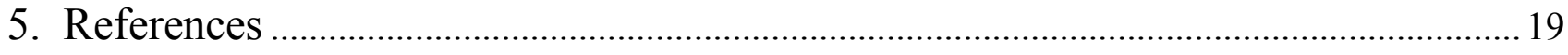




\section{Introduction}

The paper industry has had a long-standing need to better understand and to robustly control its papermaking process at the wet end, specifically, in the forming section, Fig. 1. This is motivated by the widely held belief that most of the crucial paper properties (e.g., formation) are irreversibly set by the time the slurry leaves the wet end and enters the pressing section. It is, therefore, important for effective process control to record and to analyze the pertinent paper characteristics upstream and as close to the headbox as possible.

Today's paper machines are surprisingly well instrumented. Over the past decade, a variety of intelligent instruments, including vision systems, have been developed to measure paper properties both on-line [Humphrey95, Shapiro95] and off-line [Bernie96]. While nearly all of the on-line systems operate at the dry end (see Fig. 1), research into wet-end sensors has been gaining momentum. Niemi has used a camera and illumination to determine the location and shape of the dry line at the wet end of a fourdrinier machine [Niemi94]. Whitaker has developed a nuclear backscatter gauge to measure consistency at the nip of a fourdrinier machine [Whitaker95]. Kiviranta, using stroboscopic imaging and a CCD camera, has investigated the role of table activity on formation in fourdrinier machines [Kiviranta93]. It should be noted that viewing the wet end with the aid of a strobe light as a diagnostic tool is a fairly common practice in the industry. Generally, by observing the web in this form, nonuniformities, flocculation, and the table action of the slurry on the wire may be discerned. Aidun has used high-speed imaging to investigate the dynamics of the headbox in relation to the production of streaks and other nonuniformities [Aidun97]. Nomura has also used stroboscopic imaging to show the varying nonuniformities in the sheet due to variations in headbox design [Nomura89]. The reason these researchers have focused on the wet end and, specifically, on the headbox flow conditions, is that it directly affects fiber orientation and other formation properties [Dahl88, Aidun95]. While stroboscopic imaging is established as a viable on-line technique for paper web sensing, structured lighting techniques have only been used in off-line applications, such as measuring the surface roughness of paper and board [I'Anson97]. In other application areas of computer vision, however, depth or range measurement using laser-based structured lighting is a well-established method [Vuori97, Kaplan94]. Image 
analysis and pattern recognition methodologies are also areas that have been underutilized by the paper industry researchers, especially, in the United States.

In this report, a vision system is described that utilizes stroboscopic as well as structured lighting techniques together with image processing methodologies to automatically measure and interpret the pertinent paper web parameters at the wet end. Having this capability can create both short-term and long-term changes in the paper industry and dramatically impact product quality and production yield. In the short term, the ability to characterize the web at the wet end will provide the necessary feedback to make definitive adjustments to the headbox and, hence, minimize the undesirable effects of formation variations. In the long run, the industry can expect significant advances in headbox design and control as their researchers use this same capability to better understand and quantify the headbox flow dynamics.

The presented vision system consists of two sensing subsystems. The first of these is a stroboscopic subsystem that is capable of producing high-resolution, high-quality, intensity images of the moving slurry in real-time and with $100 \%$ coverage. The other is a surface-profiling subsystem that uses a laser-based, structured lighting scheme to sense the surface topography of the slurry in real-time. This subsystem utilizes a smart, CMOS camera to accomplish fast profile calculations. The vision system also consists of image processing techniques that analyze the gathered data and determine the presence and the location of slurry inhomogeneities. These techniques are implemented on a realtime, hardware platform and use texture-based features for the detection and facet-based descriptors for the localization of slurry streaks. Presently, these techniques are applied to only the intensity images. Future work includes the application of these methods to the profile images and to the fused result of the profile and the intensity data.

The vision system hardware, in terms of the two subsystems, is presented in detail in the next section. Section 3 is dedicated to the description of the image processing algorithms and their implementations. Results obtained in the laboratory, as well as in the field are incorporated into each section individually. Section 4 includes a summary and a description of our efforts to date. 


\section{Vision system specifications}

This section presents a detailed description of the stroboscopic and the surface-profiling subsystems. Both subsystems have undergone extensive laboratory and field testing and have shown to perform satisfactorily. Images from both laboratory and field tests are included along with initial results.

\subsection{STROBOSCOPIC SUBSYSTEM}

This subsystem consists of three primary hardware modules, namely, the CCD camera, the strobed illumination source, and the real-time image acquisition and analysis platform, Fig. 2. In addition, a high-speed image storage unit was utilized to record the captured images at frame rates. These images were used to develop the image analysis algorithms, which are described in the next section. The CCD camera is a progressive scan device with 768 horizontal and 484 vertical pixels. The camera outputs images at 30 frames per second with manually-selectable electronic exposure control. Exposure control was found to be necessary (even with a strobe), because the ambient light was causing a significant background signal with large variations over the image. This effect was observed in the laboratory when the integration time was equal to the frame acquisition time of 33 ms. Reducing the exposure time down to $500 \mathrm{~ms}$ eliminated the interference even at the high ambient light levels present in a paper mill.

The strobe light is a rugged unit designed for harsh environments such as a paper mill. It is built to withstand high temperatures as well as high humidity. The particular model employed here uses a Xenon flashtube with a flash rate of 100 flashes/sec. and a maximum energy of 4.7 joules per flash. Flash duration is approximately $20 \mu \mathrm{s}$. A remote control unit allows manual control of intensity, flash rate, and phase. In order to acquire images at the time the strobe flashes, the strobe light also includes a video input that is used to synchronize the strobe to the camera. The strobe triggers on the vertical sync from the camera so that the relative time between the strobe flash and the CCD readout is fixed. The manual phase control is used to adjust the time delay between the sync signal and the firing of the strobe so that the integration time window of $500 \mu$ s. for the camera falls within the strobe flash. 
The computer system, controlling the above modules, is a PC platform that houses a PCI-based image capture and analysis board with a pipeline architecture. This board accomplishes all of the data acquisition tasks, in addition to some of the image analysis tasks. Those analysis tasks that cannot be mapped to the particular architecture of this board are assigned to the host processor of the computer. A support frame has also been designed and fabricated to hold the camera and the light during field tests. The frame can be mounted at the wet end of a paper machine and allows for the extension of the camera and the strobe light over the moving slurry for several feet.

During normal operation, the camera field of view is approximately $1 \mathrm{~m}$ wide. This width corresponds to a pixel resolution of about $1.6 \mathrm{~mm}$. A web moving at the speed of $480 \mathrm{~m} / \mathrm{min}$ will move $0.16 \mathrm{~mm}$ during the strobe time of $20 \mu \mathrm{s}$. This gives a ratio of 10 to 1 for the resolution to motion distance and ensures that the acquired image will be sharp with negligible blur due to linear motion. The described system was deployed to a paper mill and was installed on a machine producing linerboard at a speed of approximately $480 \mathrm{~m} / \mathrm{min}$. To avoid interfering with production, the frame was mounted on a catwalk seldom accessed by plant employees. The view of the paper machine from this catwalk, which was extended in the machine direction, along with a picture of the mounted apparatus are shown in Fig. 3.

The collection of images shown in Fig. 4 provides examples of the acquired images. The image in Fig.4 (a) is among the first images acquired by the stroboscopic subsystem. This is an overhead shot ( $1 \mathrm{~m}$ away) of the slurry with a field of view, the center of which was approximately $2 \mathrm{~m}$ away from the headbox and $0.8 \mathrm{~m}$ away from the edge of the web. This image, like all the others, has a resolution of $1.6 \mathrm{~mm}$ per pixel. Although some structure is visible in this image, what is immediately apparent is the specular reflection caused by the water beads, which are, in turn, produced by the deliberate shaking of the table at the wet end. While this problem could be partially mitigated by post processing the images with a median filter [Fig. 4(b)], better results were obtained when a polarizing filter was installed on the camera [Fig. 4(c)] and the output was subsequently median filtered. Note that the image in Fig. 4(d), which is the result of these two actions, shows a clear view of the streak structures that are present on the slurry. As shown in Fig. 4(e), it is interesting to see how a small deposit (encircled) attached to the lip of the headbox can contribute to the generation of web streaks. Finally, the image in Fig. 4(f), which is an image of fiberless water, 
shows a clear distinction between the visual appearance of water alone and that of slurry, with the former exhibiting more homogeneity. $1 \mathrm{~m} 1.2 \mathrm{~m} \tilde{\square} 7$

\subsection{SURFACE-PROFILING SUBSYSTEM}

This subsystem is designed to capture the surface topography of the slurry at the wet end of a paper machine. It uses a structured lighting scheme and is composed of two primary modules, namely, the line-projecting laser source and the CMOS, smart camera (see Fig. 5). The 100-mW, 830-nm, infrared, diode laser with optics to spread the beam into a plane of light is placed directly above the web. The light plane is perpendicular to the motion direction and is parallel to the cross direction. The CMOS camera is mounted at an angle to the laser, viewing the web as it moves underneath. The 830-nm laser is invisible to the eye, but the silicon in the camera is highly sensitive to energy at this wavelength. An infrared pass filter is used to exclude visible light to the camera, so that external lighting does not interfere with the algorithm used to locate the laser line position. The laser projects a single line of light onto the surface at a fan out of 45 degrees. With the camera at an angle $\theta$ from the laser, the change in image height, as seen by the camera, is related to the actual depth change through $d y=\sin \theta d z$, where $d y$ is the change in the $y$ coordinate relative to the camera and $d z$ is the change in depth (Fig. 6). This expression demonstrates that the resolution with which the depth is measured increases as $\theta$ approaches its maximum value of 90 degrees. However, larger angles can potentially cause occlusions by surfaces that are not in the laser light plane. This, in turn, gives rise to broken laser lines and, therefore, missing data in the collected images. Generally, a satisfactory compromise can be reached if this angle is set to 45 degrees, producing a sensitivity of approximately $70 \%$ of the maximum.

During normal operation, the laser sheet of light intersects the surface of the slurry, producing an irregular line. The smart camera acquires an image of this line and proceeds to detect its location through a simple thresholding procedure. Once the laser line has been detected, the next step is to estimate the center of this line within each column of the image. As discussed before, the estimated centers or, more specifically, their displacement from a reference point, correspond to the surface profile of the slurry. Two algorithms for the estimation of the laser line centers were implemented, and their performances were compared. Before presenting a brief description of these algorithms, it should be pointed out that the advantage of employing a smart (i.e., programmable) camera is that 
the implemented algorithms are executed on board the camera, resulting in processing speeds in the order of hundreds of lines per second.

The first of these algorithms, referred to as the binary centroid algorithm, is a straightforward technique that operates as follows. Within each column, the intensity values of the contained laser line are thresholded to produce a one-dimensional blob. Then, the surface profile of the object in each location is computed as the centroid of the obtained blob according to

$$
z=\frac{1}{A} \sum_{i} r_{i}
$$

where $r_{i}$ represents the coordinate (row position) of the pixels whose intensity values are above the threshold, and is the total number of such pixels.

The second algorithm, referred to as the weighted centroid algorithm, is a more sophisticated technique and one that is computationally more complex [DePiero96]. As was the case above, the procedure that is to be described is repeated for each column of the laser line image. The distinct characteristic of the weighted centroid algorithm is a smooth mapping function, $P\left(g_{i}\right)$, that is used to represent the likelihood that a pixel with intensity $g_{i}$ is a member of the laser line. This is in contrast to the binary centroid algorithm, in which the decision of whether or not a pixel is a member of the laser line is one that is crisp rather than fuzzy. In this work, a truncated error function (erf) is chosen for the smooth mapping function. The shape of the Gaussian, which is integrated to compute the values of $P\left(g_{i}\right)$, is chosen so that 3 standard deviations below the mean are those intensity values that clearly belong to the background. Then, the surface profile of the object in each column is computed as follows:

$$
z=\frac{1}{S} \sum_{i} P\left(g_{i}\right) r_{i},
$$

where $S=\sum P\left(g_{i}\right)$. The surface profile information estimated in this fashion, though computationally more intensive, is far more robust than the binary centroid algorithm. 
To test the apparatus and the algorithms described above, a scene comprising rows of cotton balls glued to the transported paper was fabricated in the laboratory [Fig. 7(a)]. This test scene was scanned by our surface-profiling system at various web speeds. The 3-D plot of Fig. 7(b) represents the estimated surface profile of the test scene at $30 \mathrm{~m} / \mathrm{min}$. The 2-D contour plots in Figs. 7(c)-7(e) give an interesting view of the rows of cotton balls at 15,30 , and $150 \mathrm{~m} / \mathrm{min}$, respectively. Note how the lengths of the rows are shortened as the number of samples in the direction of motion decreases with increased speed of the web. The surface-profiling subsystem was also deployed and installed on the wet end of the paper machine, shown in Fig. 3, to capture the surface topography of the slurry. The main challenge in sensing the web in this manner was that upon contact with the slurry, the laser line became diffused, making it difficult to accurately discern its displacement in the $y$ direction. Although this difficulty led to a decreased resolution in the depth measurements, the overall topography of the slurry was nevertheless captured, Fig. 8. In future work, an anamorphic lens could be installed on the camera to increase the resolution of the measurements in the $y$ direction without compromising the length of our field of view.

In addition to the described subsystems, the vision system also consists of image processing techniques that analyze the gathered data and determine the presence and the location of slurry inhomogeneities. These algorithms are described next.

\section{Image analysis for streak detection and localization}

Two algorithms for the detection and the localization of slurry streaks have been implemented and thoroughly tested using both synthesized and real images. The first of these is a texture-based algorithm that produces a single numeric output indicating the presence or absence of the streaks in the field of view. The second algorithm, based on the facet model, aids in accurately localizing the streaks. These techniques are implemented on a real-time, hardware platform with a pipeline architecture. Presently, these algorithms are applied to only the intensity images. Future work will include the application of these methods to the profile images and to the fused result of the profile and the intensity data. 


\subsection{TEXTURE-BASED STREAK DETECTION ALGORITHM}

The essence of this algorithm is to detect that which disturbs the global homogeneity of the slurry images. To accomplish this, two techniques (i.e., discrete wavelet transform and correlation dimension) are combined to measure the global homogeneity of the input images. Subsequently, a threshold is employed to distinguish between the absence and the presence of slurry streaks. The introduced scheme of homogeneity measurement has been utilized successfully in similar applications in the past [Sari-Sarraf99]. Below are described the two techniques that make up this algorithm.

By wavelet transform, we are in fact referring to a specific class of the 2-D discrete wavelet transform called the multistage wavelet representation (MSWAR) [Sari-Sarraf97]. The primary difference between MSWAR and the standard discrete wavelet transform is that the generated signals (or images) in the former case remain at full resolution, whereas in the latter case their resolution is reduced (through decimation) with each iteration of the transformation process. For object recognition and feature extraction (as with this work), this distinction becomes especially significant because loss of resolution means that measurements must be made with fewer data points.

The MSWAR of a 2-D image $f(x, y),(x, y)=1,2, \ldots, N$, with levels of scale reduction is a set of $(3 M+1)$ images. These are the detail images at all levels of scale reduction:

- $f_{d 1}^{j}(x, y)$ (contains all but vertical edges),

- $f_{d 2}^{j}(x, y)$ (contains all but horizontal edges),

- $f_{d 3}^{j}(x, y)$ (contains all but horizontal or vertical edges), for $j=1,2, \ldots, M$,

plus $f(x, y)$ 's blurred version at the lowest scale level, $f^{M}(x, y)$. An efficient algorithm for the generation of these images has been derived in [Sari-Sarraf97], and is given below for easy reference. 
1. Given a low-pass and a high-pass filter, and assuming that these filters are represented as column vectors $L P$ and $H P$, respectively, generate four 2-D kernels as follows: $L P(L P)^{t}, H P(L P)^{t}, L P(H P)^{t}, H P(H P)^{t}$, where $(.)^{t}$ denotes vector transposition.

2. For $j=1,2, \ldots, M$,

3. For $x=0,1, \ldots, N-1$,

4. For $y=0,1, \ldots, N-1$,

5. Allocate $u$ row pointers, $p_{0}, p_{1}, \ldots, p_{u-1}$, and $u$ column pointers $q_{0}, q_{1}, \ldots, q_{u-1}$, where $u$ indicates the support of the selected filters.

6. Initialize the above pointers as follows:

$$
\begin{aligned}
& p_{0}=x, p_{1}=p_{0}+2^{j-1}, \ldots, p_{u-1}=p_{u-2}+2^{j-1} \\
& q_{0}=y, q_{1}=q_{0}+2^{j-1}, \ldots, q_{u-1}=q_{u-2}+2^{j-1}
\end{aligned}
$$

7. Convolve the generated kernels with the elements of the signal $f^{(j-1)}$, where $f^{0}=f(x, y)$, as addressed by the above pointers. The results are the $(x, y)^{\text {th }}$ elements of the four output signals $f^{j}, f_{d 1}^{j}, f_{d 2}^{j}$, and $f_{d 3}^{j}$, respectively.

8. Next $y$.

9. Next $x$.

10. Next $j$.

The choice of the above-mentioned low-pass and high-pass filters is application dependent. Thus far in this work, we have utilized Daubechies' filters [Daubechies88] for their compact support and orthogonality. An example of the application of this algorithm to an image of the slurry is shown in Fig. 9. Note that, due to the presence of a streak, the homogeneity of the output image in Fig. 9(b) is diminished. Simply put, the wavelet transform and MSWAR, because of their multiscale nature, allow a choice of the appropriate scale of operation, for which the desired image features are accentuated and the image clutter is attenuated. Furthermore, MSWAR allows for an orientationselective analysis. This is important in our application, because the streaks always occur in the 
direction of motion. We now present a fractal-based measure that is used to quantify the global homogeneity of the detail images, specifically, $f_{d 1}^{j}(x, y)$, as produced by the wavelet transformation of the slurry images.

Fractal-based measurements, such as the fractal dimension, lacunarity, and the correlation dimension, have been utilized extensively, with varying degrees of success, for quantifying surface characteristics of texture images. In this work, two new measurements, which are derived from the correlation dimension, are utilized [Brzakovic94]. The first of these is a local measurement that quantifies the surface roughness, while the second gives a measure of the surface homogeneity in a global sense. Let us start by introducing the correlation dimension.

Let a gray level image, $f(x, y)$, be represented by a point in 3 -D space as $\vec{X}_{i}[x, y, f(x, y)]$. The correlation dimension, as introduced by Grassberger and Procaccia [Grassberger83], is defined as

$$
v=\lim _{\varepsilon \rightarrow 0} \frac{\log [C(\varepsilon)]}{\log [\varepsilon]}
$$

where $\varepsilon$ denotes scale. The correlation sum, $C(\varepsilon)$, is given as

$$
C(\varepsilon)=\lim _{N \rightarrow \infty} \frac{1}{N^{2}} \sum_{\substack{i, j=1 \\ i \neq j}}^{N} \Theta\left(\varepsilon-\left\|\overrightarrow{X_{i}}-\overrightarrow{X_{j}}\right\|\right)
$$

where $N$ is the total number of points in the set; $\Theta(x)$ denotes the unit step function; and $\left\|\overrightarrow{X_{i}}-\overrightarrow{X_{j}}\right\|$ is the Euclidean distance between vectors $\overrightarrow{X_{i}}$ and $\overrightarrow{X_{j}} \cdot$ Generally, the correlation dimension is estimated as the slope of the line that is fitted to the data points $\{\log (\varepsilon), \log [C(\varepsilon)]\}$. In this work, however, two measurements are derived directly from the correlation sum.

The first of these reflects the local roughness of the input image surface and is given as 


$$
R(m, n)=\sum_{\varepsilon=1}^{\varepsilon_{u}} C^{2}(\varepsilon, m, n)
$$

where $\varepsilon_{u}$ is the upper limit for $\varepsilon$, and $C(\varepsilon, m, n)$ is the correlation sum computed within nonoverlapping subregions of the input image. The second measurement quantifies global image homogeneity and is computed as follows:

$$
V=\frac{1}{Q} \sum_{m} \sum_{n}[R(m, n)-M]^{2},
$$

where

$$
M=\frac{1}{Q} \sum_{m} \sum_{n} R(m, n),
$$

and $Q$ is the total number of subregions into which the input image is divided. Given the above expressions, the following statements can be made. High values of $R(m, n)$ signify high correlation among the pixel values in the subregion (corresponding to a smooth surface), while low values of $R(m, n)$ indicate a rough surface. Furthermore, small values of $V$ (i.e., surface is either mainly rough or mainly smooth) denote a homogeneous image.

Combining MSWAR with the above measurements provides a powerful tool for quantifying the global homogeneity of texture images. By applying the local roughness and global homogeneity measures to the output of MSWAR (specifically, the detail images), one can quantify these features in a scale-dependent, orientation-selective fashion. Images in Fig. 10 show how the appearance and disappearance of a streak captured in four consecutive frames of the slurry is quantified with the proposed homogeneity measure. As the streak appears in the field of view, the computed value of increases, indicating that the homogeneity of the slurry image is disrupted. On the other hand, the measured values decrease as the streak leaves the field of view. Furthermore, the computed values of homogeneity in the presence and absence of streaks differ by an order of magnitude, making the task of setting a threshold an easy one. Plots of Fig. 11 further illustrate the efficacy of the proposed 
homogeneity measure in successfully detecting slurry streaks. Conducting a test that included over 500 images, it was verified that every local maximum (i.e. $V \geq 1500$ ) in the plot of Fig. 11(a) did, indeed, correspond to the presence of a streak in the corresponding image of the slurry. The plot of Fig. 11(b) demonstrates how the paper web at the wet end becomes more homogeneous when the slurry is abruptly switched to water. Paper machine operators take this action deliberately when there is a break in the web downstream. Images of Fig. 4(d) and 4(f) show a clear distinction between the apparent homogeneity of water alone and that of slurry.

Real-Time Implementation of Streak Detection Algorithm. Development has been completed for the real-time implementation of the global homogeneity measurement using MSWAR and the local roughness variance, as described above. The algorithm is implemented using a dedicated pipeline processing module and a general-purpose computer. At present, the MSWAR algorithm runs on the pipeline processor, while the texture calculations are performed on the general-purpose computer. Through a graphical user interface, a real-time plot of the global homogeneity is displayed for up to two separate regions in the acquired image. The overall frame rate achieved on the existing hardware is 10 frames per second for a 256 by 256 image. Most of this time is required by the texture calculations, since the computation of MSWAR is accomplished at a $40-\mathrm{MHz}$ pixel rate. This frame rate is scalable to 30 frames per second with currently available processors. Significant additional improvements in speed are also possible by implementing more of the algorithm on the dedicated hardware.

\subsection{FACET-BASED STREAK LOCALIZATION ALGORITHM}

While the texture-based algorithm, described in the previous section, is an effective tool for the detection of slurry streaks, it cannot fulfill the demand for an accurate localization of these structures. To meet this demand, we have designed and implemented an algorithm that not only indicates the location, size, and the orientation of the streaks, but it also characterizes the surface topography of the slurry. This algorithm, which is based on the facet model [Haralick92], consists of three sequential steps: (1) background subtraction, (2) facet model generation, and (3) morphological filtering. This algorithm is intended to be applied to the output of the surface- 
profiling subsystem but thus far, has only been used with intensity images of the slurry. In what follows, each of the above four steps are described in sufficient detail.

Background Subtraction. The intensity images that are collected online using the stroboscopic subsystem often suffer from nonuniform illumination, which can adversely affect the streak localization process. To overcome this problem and to enhance the contrast of the slurry structures, we employ the background subtraction technique [Sternberg83]. This technique consists of a background removal step, in which the slowly varying portion of the image is separated and then subtracted from the original image. The histogram of the resulting image is subsequently stretched to enhance the more sharply defined features of interest. The background subtraction is implemented using the rolling ball method. This method is based on gray-scale morphology and amounts to a morphological opening of the 3-D graph of the input image (intensity values represent the third dimension) with a spherical structuring element. The size of the foreground features that are to be excluded from the background is controlled by varying the radius of the structuring element. After the background image is subtracted, the remaining image generally has less dynamic range than the original. A histogram stretch is applied in which the tails are truncated to expand the dynamic range and enhance the features of interest that remain. An example of the application of this methodology to an image of slurry is shown in Fig. 12.

Facet Model Generation. The facet model, or more specifically, the topographic primal sketch [Haralick92] consists of labeling and grouping the underlying image surface patches into categories such as peaks, pits, ridges, ravines, and hillside convexities and concavities. The facet model principle assumes that the image can be characterized as a piecewise-continuous surface. To carry out the characterization, a model is specified that adequately describes the general form of the surface in the neighborhood of any pixel. The commonly used models include piecewise constant, piecewise linear, piecewise quadratic, and piecewise cubic. Assuming, for now, that the images are not degraded by noise or geometrical transformations (e.g., defocusing), and utilizing one of the above-mentioned forms, one must first estimate the model parameters for all the pixels. Subsequently, these estimates can be utilized in a variety of ways, including the labeling of every pixel into one of a variety of topographic structures. 
In this work, we compute the best-fit (in a least-squares sense) cubic facet for each pixel and its neighborhood in the input image. Determined experimentally, the neighborhood size is chosen to be 13 by 13 , and the cubic facet is set to be a cubic polynomial of the form

$$
f(x, y)=k_{1} x+k_{2} y+k_{3} x^{2}+k_{4} x y+k_{5} y^{2}+k_{6} x^{3}+k_{7} x^{2} y+k_{8} x y^{2}+k_{9} y^{3}+k_{10}
$$

where $f(x, y)$ is the gray level value at pixel location $(x, y)$ whose neighborhood is to be fitted. Once the10-element vector of coefficients has been estimated for each pixel, the gradient and the Hessian of the facets are computed. Finally, the topographic labeling of each pixel is accomplished by determining whether various derived quantities are negative, zero, or positive. These quantities include the gradient magnitude, the largest and the smallest eigenvalues of the Hessian, and two directional derivatives. For details on the labeling process, the reader is referred to [Haralick92]. The synthetic image of Fig. 13 demonstrates the efficacy of this approach in characterizing the underlying surface topography. The computation of the facet model coefficients and the subsequent topographic labeling of the image pixels were performed on hundreds of slurry images. It was then determined that, based on the manner in which the paper web was illuminated, three of the labels (i.e., hillside convex, hillside concave, and hillside saddle) yield the best characterization of the streaks [see Fig. 14(b) for an example].

Morphological Filtering. Resulting from the last two steps is a binarized image that contains all the pixels in the input slurry image corresponding to three topographic labels mentioned above. These pixels represent the slurry streaks, as well as other less interesting features that are smaller in size and exhibit little or no directional preference. Hence, morphological processing is used towards the filtering of these noise features. This includes a closing with an elongated (in the horizontal direction) structuring element, followed by an opening [Fig. 14(c)]. The objects resulting from the morphological processing can then be enclosed within a minimum bounding box and be characterized according to their size, orientation, elongation, etc. [see Fig. 14(d)].

Real-Time Implementation of Streak Localization Algorithm. A real-time facet model implementation has been developed for computing the surface feature parameters of the slurry at $100 \%$ web coverage. This implementation is on a combination of dedicated pipeline processing 
hardware and a general-purpose computer. Fig. 15 shows block diagrams of the design. The cubic polynomial approximation consists of ten terms with coefficients $k_{1}$ through $k_{10}$. Each coefficient is calculated by convolving the input image with a mask. The mask is predetermined to compute the least square fit of the Chebyshev polynomials to the input image. On the pipeline processing hardware, dedicated convolution modules are used for this computation. These computations are performed at $40 \mathrm{MHz}$, so that a 256 by 256 image will require $1.6 \mathrm{~ms}$ for each coefficient calculation. With a100-point convolver, two convolutions can be performed at the same time with up to a 7 by 7 convolution mask. The total time for the coefficient calculations is approximately 8 ms. Larger convolutions with a 9 by 9 mask would require $16 \mathrm{~ms}$. After convolution, the coefficients are stored in memory and are used in the next step of the feature calculation pipeline. Using arithmetic blocks and look-up tables in hardware, the gradient, the gradient magnitude, the eigenvalues and eigenvectors of the Hessian are calculated. From these quantities the facet model feature values are determined. The frame rate achieved using the 7 by 7 masks and 256 by 256 images is 30 frames/sec. The required processing rate is determined by the requirement for $100 \%$ inspection of the slurry at the maximum operating speed of the paper machine. A frame rate of 30 frames/sec is sufficient to provide this coverage for web speeds up to $1500 \mathrm{ft} . / \mathrm{min}$. Higher web speeds can be achieved through the addition of a second pipeline processor and a higher frame rate camera. The described facet-based, streak localization algorithm was successfully applied to hundreds of slurry images, which were captured on-line using the stroboscopic subsystem; see Fig. 14.

\section{Summary}

In this report, we have described a vision system that accomplishes the novel task of characterizing the slurry streaks at the wet end of a paper machine. The vision system is a combination of state-ofthe-art hardware modules plus a unique combination of algorithmic procedures that together detect and localize the slurry nonuniformities. Specifically, the system is capable of monitoring the paper slurry upon its exit from the headbox and alerting the operators of any event that disrupts the

otherwise homogeneous background. Essentially, the system acquires both intensity and topographic information from the scene and uses texture-based features for the detection, and facetbased descriptors for the localization of the nonuniformities. In addition to being tested in a 
laboratory environment, a prototype of this system has been deployed to a paper mill and its performance has been evaluated under realistic conditions. Installed on a fourdrinier paper machine, running at $480 \mathrm{~m} / \mathrm{min}$ and producing linerboard material, the vision system was able to monitor an approximately 1-meter-wide area and to successfully detect and localize slurry streaks. Two main areas have been investigated. The first of these is the application of the facet-based streak localization algorithm to the topographic data, as gathered by the surface-profiling subsystem. As mentioned above, thus far, this algorithm has only been applied to the intensity images of the stroboscopic subsystem. The second area, which requires considerable attention, is the determination of the degree of correlation between the appearance of streaks in the slurry and the ultimate quality of the manufactured paper. This study requires a controlled field experiment in which our on-line measurements are compared against the standardized measurements that the industry currently uses to assess paper quality. 


\section{References}

C.K. Aidun, "Hydrodynamics of Streaks on the Forming Table," Tappi Journal, August 1997.C.K. Aidun, and A.E. Kovacs, "Hydrodynamics of the Forming Section: The Origin of Nonuniform Fiber Orientation," Tappi Journa1, 78, No. 11, November 1995.

J. -P. Bernie, and W.J.M. Douglas, "Local Grammage Distribution and Formation of Paper by Light Transmission Image Analysis,” Tappi Journal, 79, No. 1, January 1996, pp. 193-202.

C.J. Biermann, Handbook of Pulping and Papermaking, 2nd Edition, Academic Press, New York, 1996.

D. Brzakovic, and H. Sari-Sarraf, "Automated Inspection of Nonwoven Web Materials: A Case Study,” Proc. of SPIE's Symposium on Electronic Imaging, San Jose, CA, February 1994.

H. Dahl, H. Holik, and E. Weisshuhn, "The Influence of Headbox Flow Conditions on Paper Properties and Their Constancy," Tappi Journal, February 1988, pp. 93-98.

Daubechies, "Orthogonal Bases of Compactly Supported Wavelets," Comm. Pure and Appl. Math, 41, 1988, pp. 909-996.

F.W. DePiero, and M.M. Trivedi, "3-D Computer Vision Using Structured Light: Design, Calibration and Implementation Issues," Advances in Computers, 43, 1996.

P. Grassberger, and I. Procaccia, "Characterization of Strange Attractors," Phys. Rev. Letters, 50,No.5, 1983, pp. 346-349.

R.M. Haralick, and L.G. Shapiro, Computer and Robot Vision, Volume I, Addison-Wesley, New York, 1992.

K. Humphrey, Image Analysis, Pira International, 1995.S. I'Anson, "3-D Laser Surface Profiling of Paper and Board," Paper Technology, 38, No. 2, March1997, pp. 43-49.

H. Kaplan, "Structured Light Finds a Home in Machine Vision,” Photonics Spectra, January 1994.

A. Kiviranta, "The Role of Table Activity in Fourdrinier Forming," Canadian Pulp and Paper Association79th Annual Meeting Technical Section, Montreal, Canada, January 1993.

A.J. Niemi, and C.J. Backstrom, "Automatic Observation of Dry Line on Wire for Wet End Control of the Paper Machine," Pulp and Paper Canada, 95, No. 2, February 1994, pp. 27-30.

T. Nomura, K. Wada, and T. Shimizu, "High Consistency Sheet Forming," Tappi Journal, January1989, pp. 115-122. 
H. Sari-Sarraf, and D. Brzakovic, "A Shift-Invariant Discrete Wavelet Transform," IEEE Trans. On Signal Processing, 45, No. 10, October 1997, pp. 2621-2626.

H. Sari-Sarraf and J. S. Goddard, "Vision System for On-Loom Fabric Inspection," IEEE Trans. On Industry Applications, 35, No. 6, December 1999, pp. 1252-1259.

S.I. Shapiro, and R.H. Shearin, "On-Line Sensors: Exploring the Trends," Tappi Journal, 78, No. 9,September 1995, pp. 83-84.

S.R. Sternberg, "Biological Image Processing”, Computer, 1983, pp. 22-34.

T.A. Vuori, and C.L. Smith, "Three-Dimensional Imaging System with Structured Lighting and Practical Constraints," Journal of Electronic Imaging, 6, No. 1, January 1997, pp. 140-144.

M. Whitaker, "Optimising Formation Through Consistency Measurement - a Wet End Revolution," Paper Technology, 36, no. 2, March 1995, pp. 22-26. 


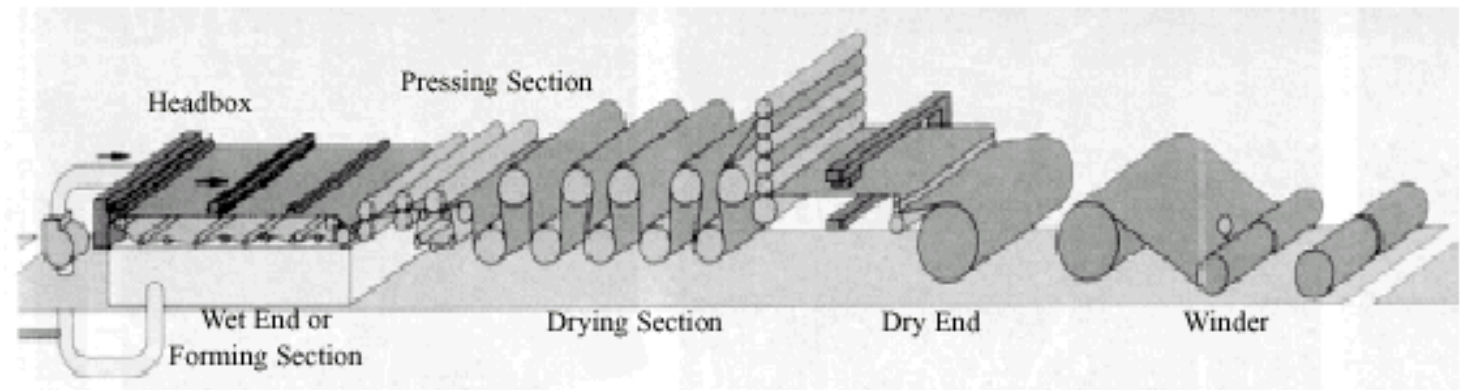

Figure 1. Layout of a typical fourdrinier paper machine. Picture reproduced from [Biermann96].

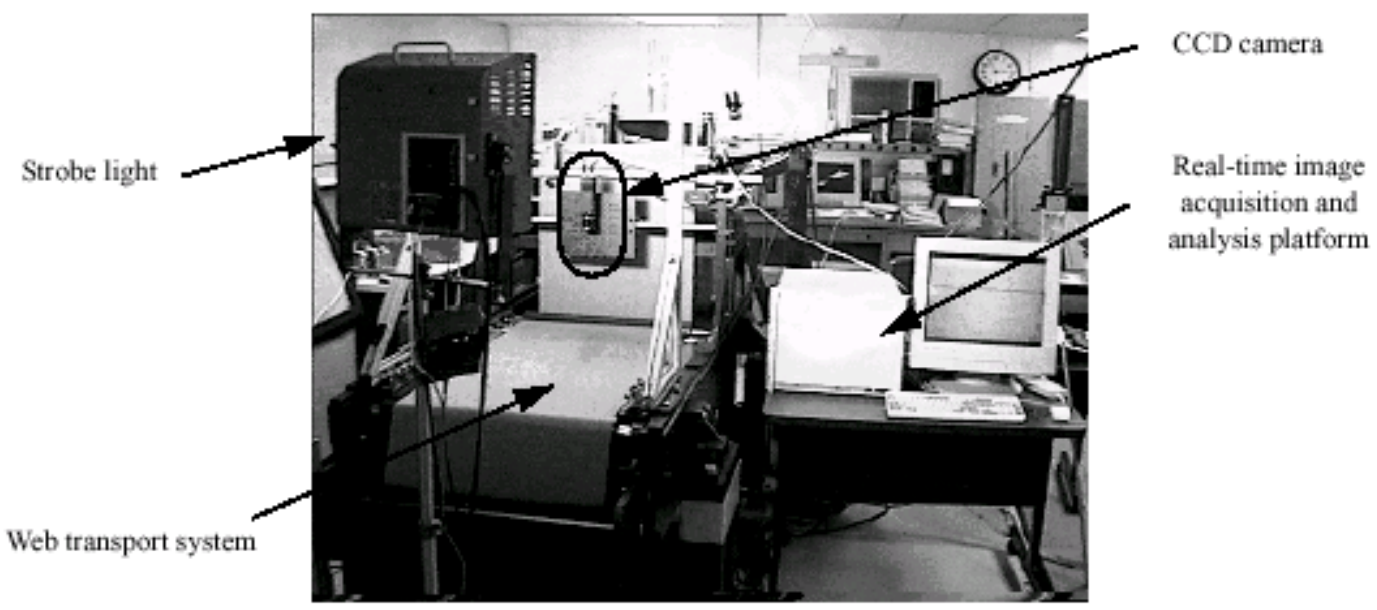

Figure 2. Laboratory setup of the stroboscopic subsystem and its primary components.

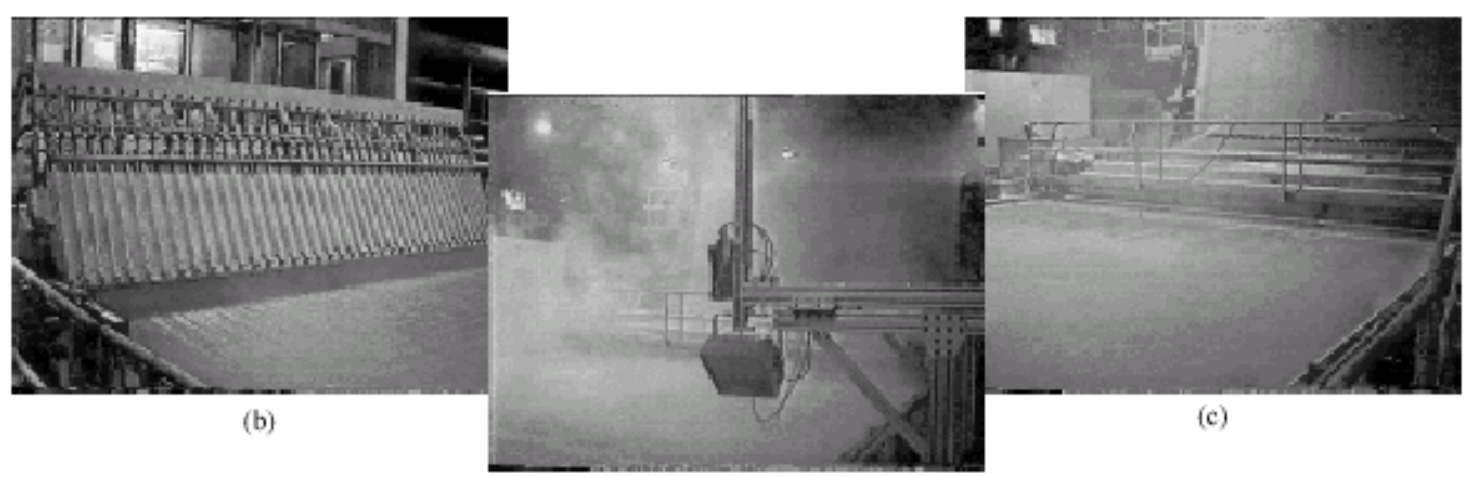

(a)

Figure 3. Field-deployed stroboscopic subsystem. The system shown in (a) is installed at the wet end of the paper machine between the headbox in (b) and the pressing section in (c). 


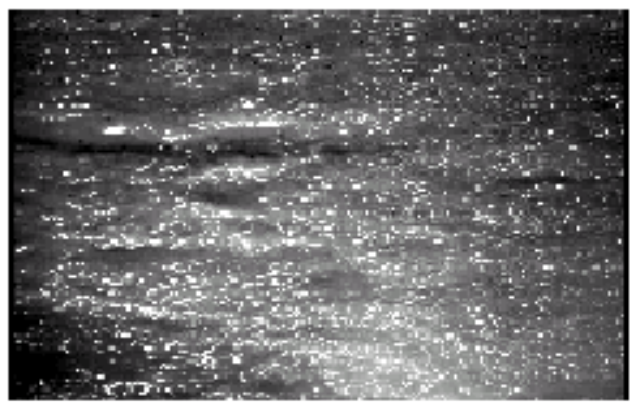

(a)

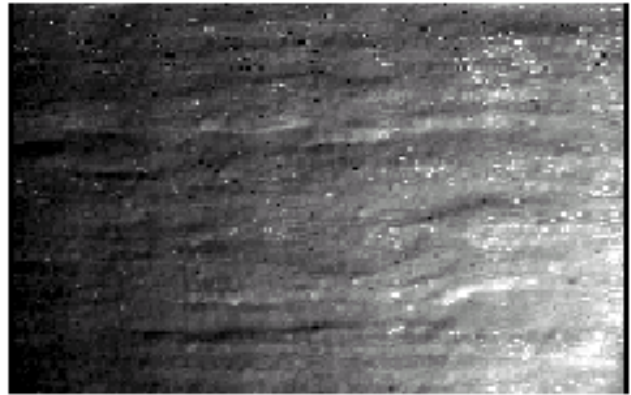

(c)

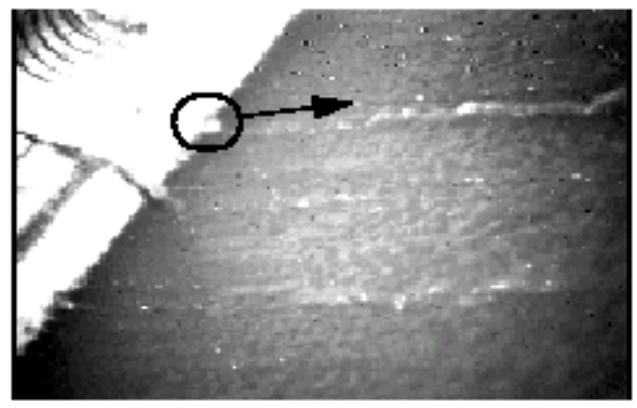

(e)

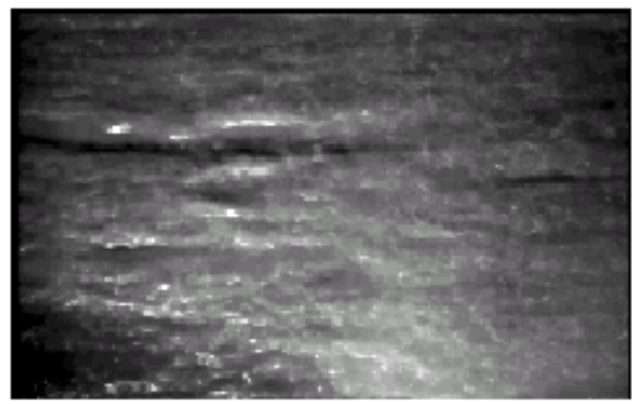

(b)

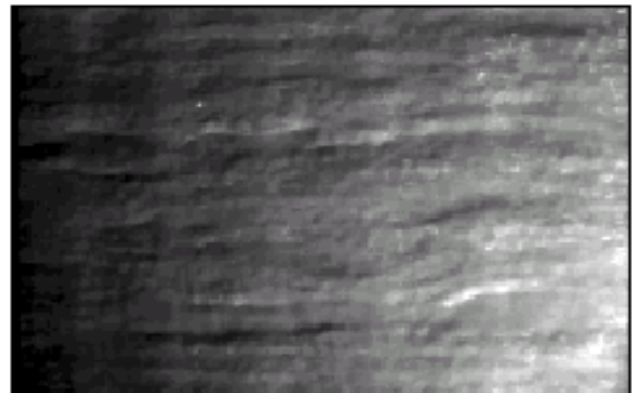

(d)

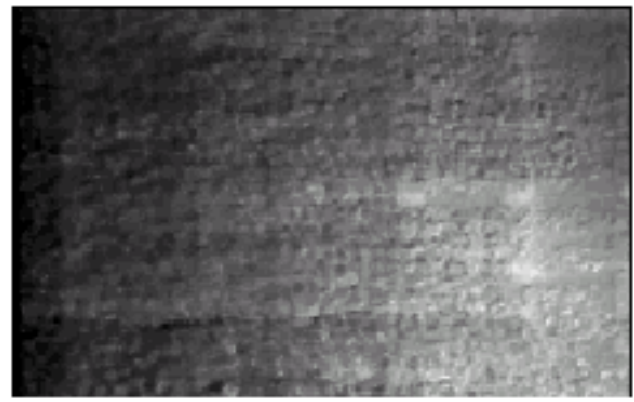

(f)

Figure 4. Example of images collected during a field test of the stroboscopic subsystem. In all images, the direction of motion is from left to right. See text for more details. 


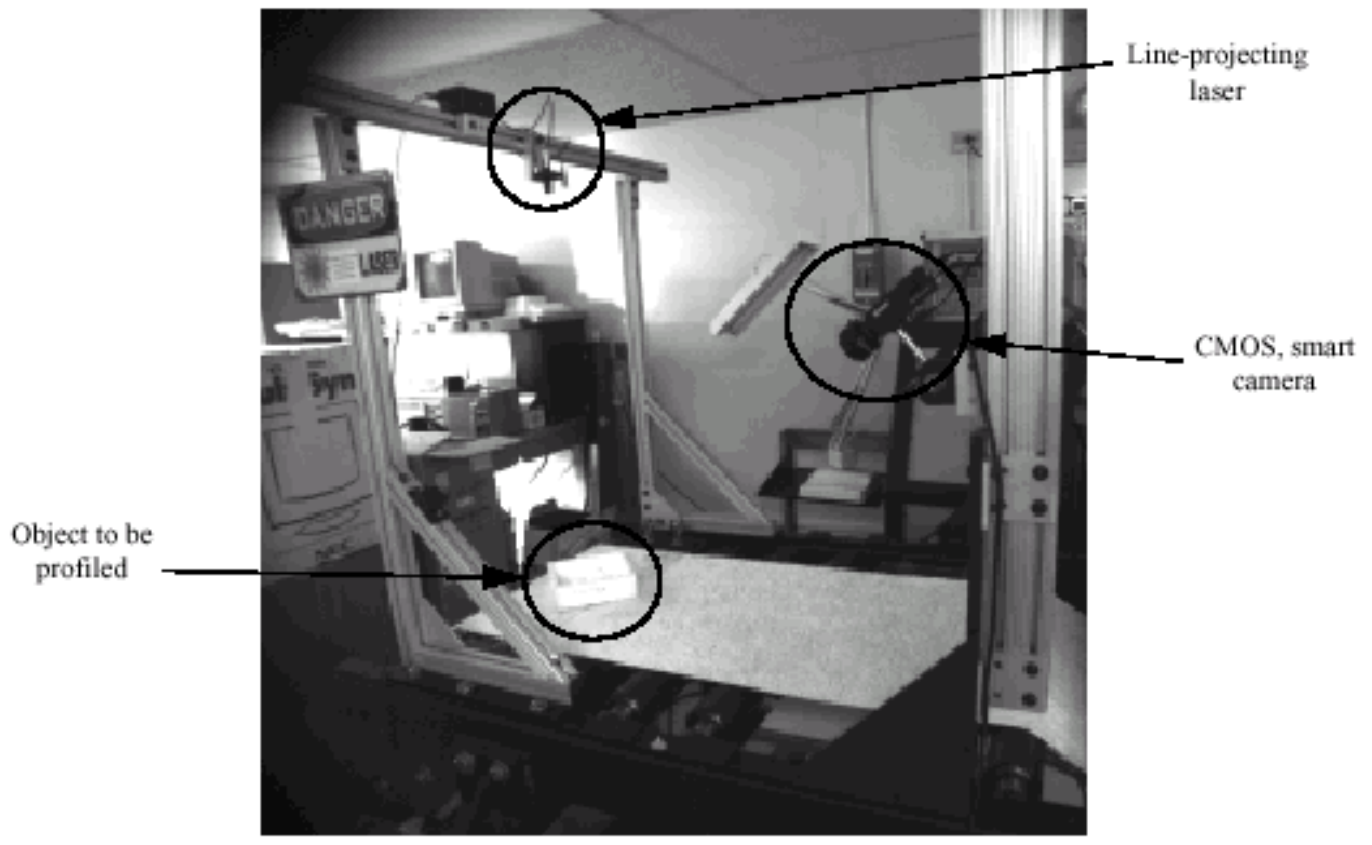

Figure 5. Laboratory setup of the surface-profiling subsystem and its primary components.

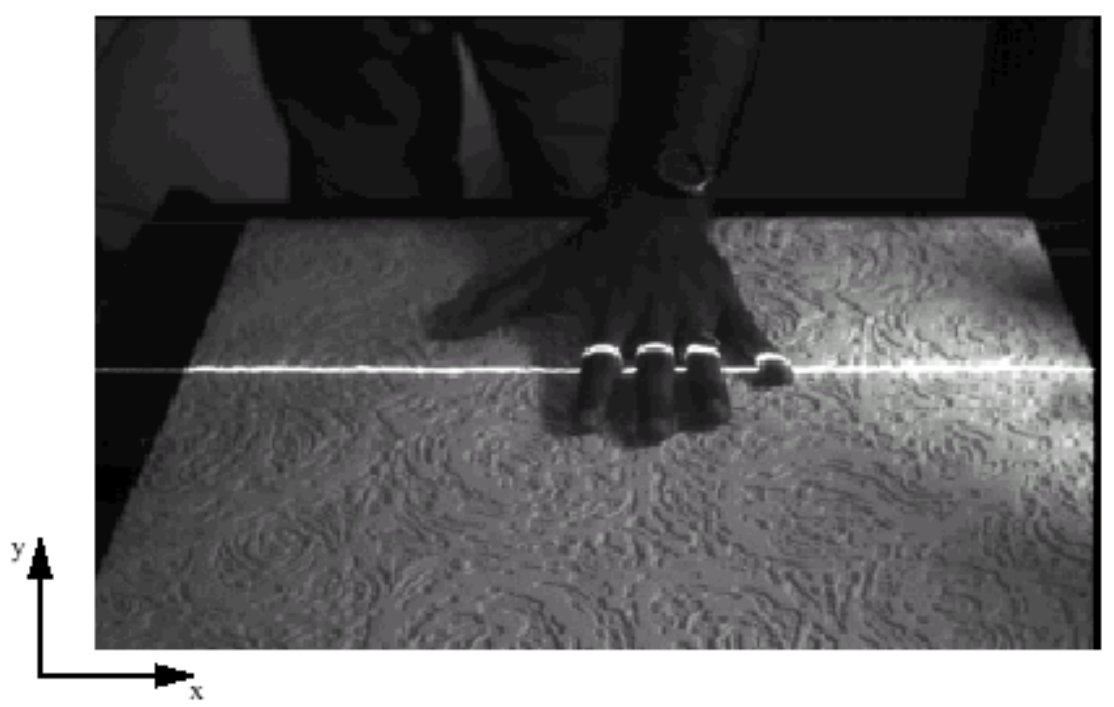

Figure 6. The bright horizontal line in the center of the image is the laser line as seen by the CMOS camera. Note how its displacement in the $y$ direction relates to the depth of the fingers. 


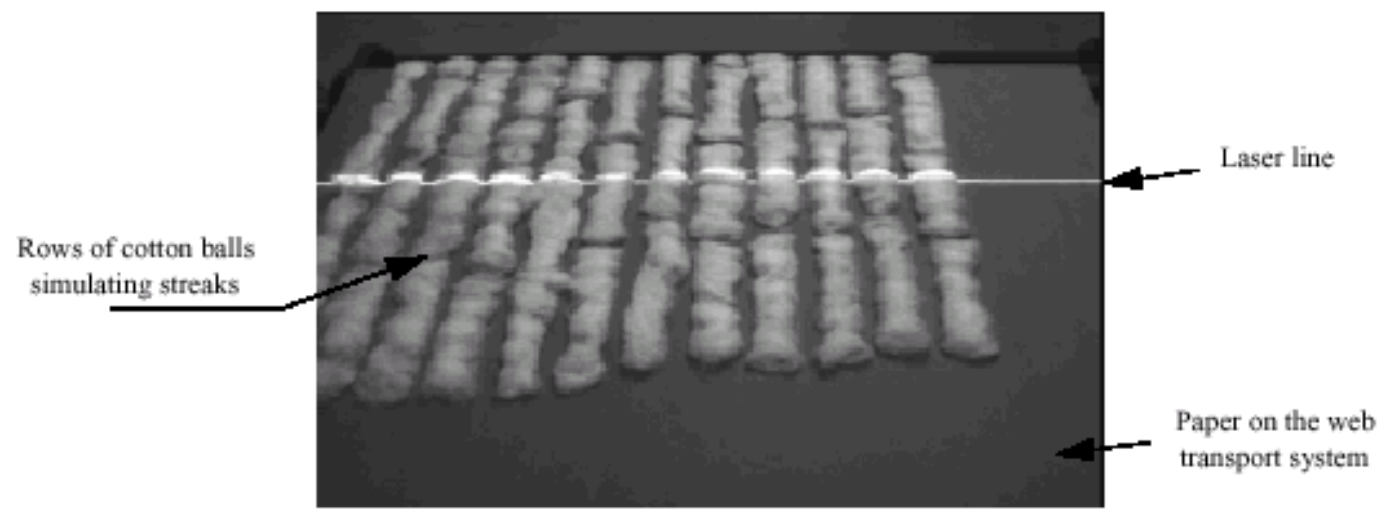

(a)

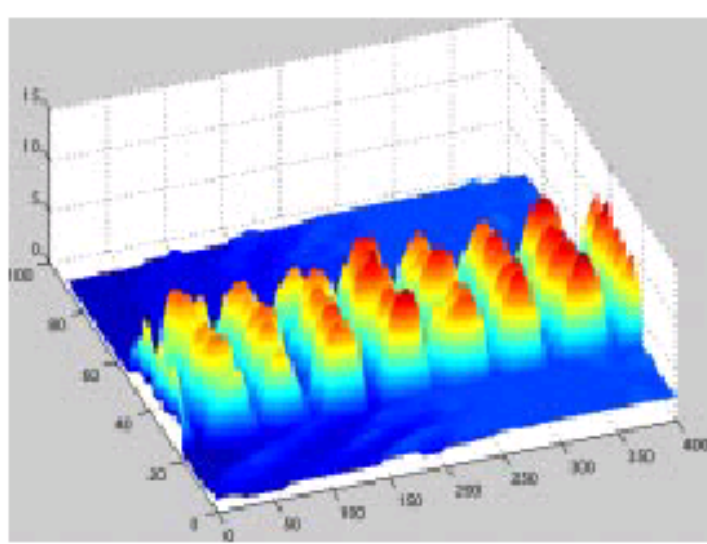

(b)

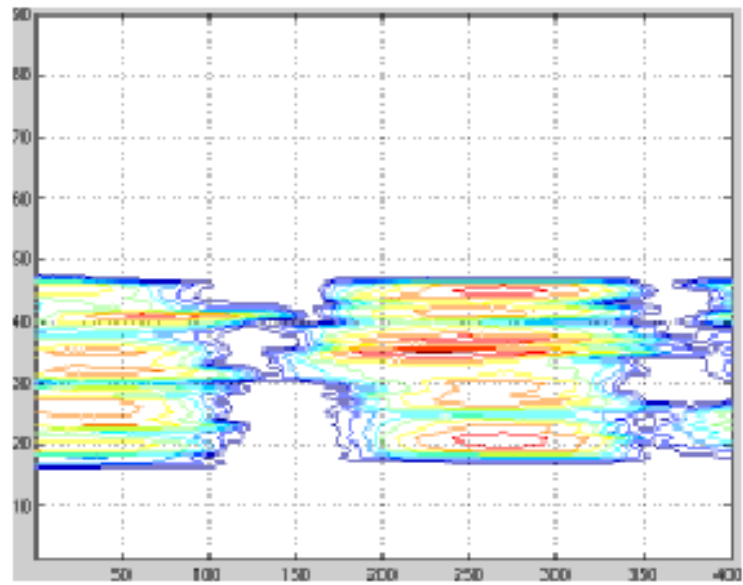

(d)

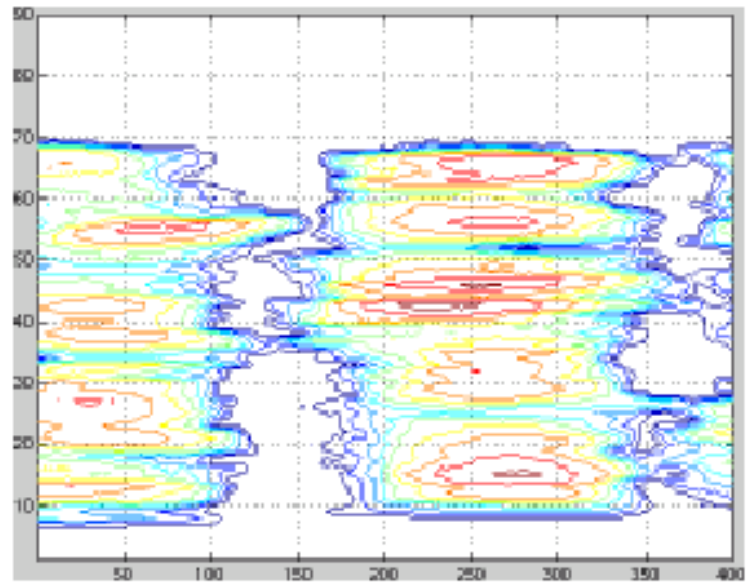

(c)

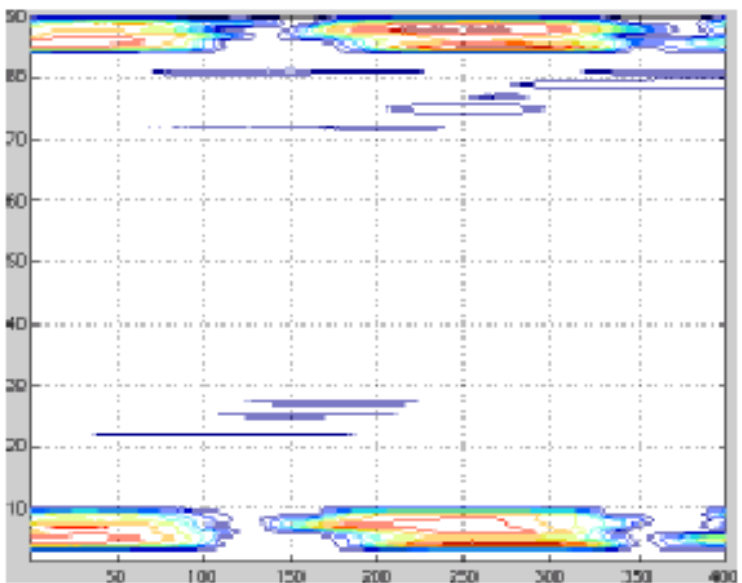

(e)

Figure 7. (a) Test scene sensed by the surface-profiling subsystem. (b) Surface profile of the scene. (c), (d), and (e) Surface profiles of the test scene scanned at 15,30 , and $150 \mathrm{~m} / \mathrm{min}$, respectively, and represented as contour plots. 


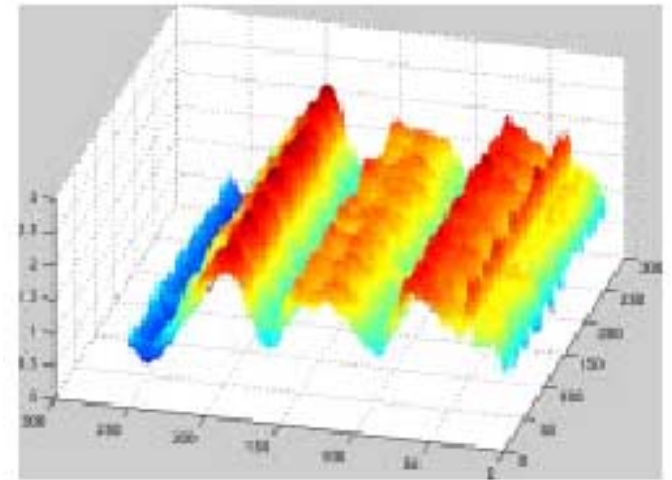

(a)

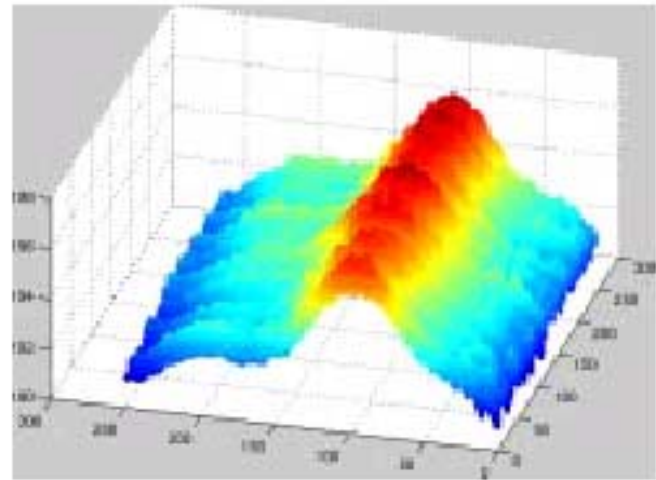

(b)

Figure 8. Captured topography of slurry using the surface-profiling subsystem with (a) a 30-mm, and (b) a 75mm lens.

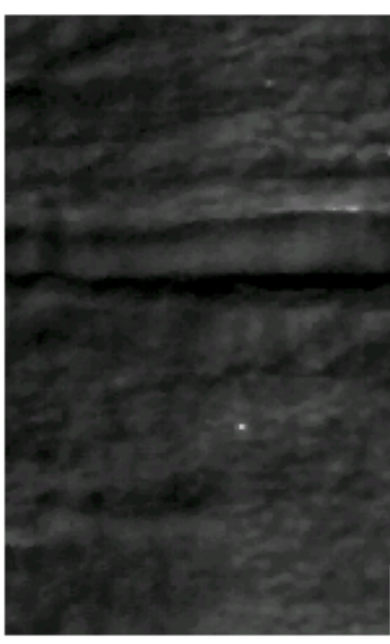

(a)

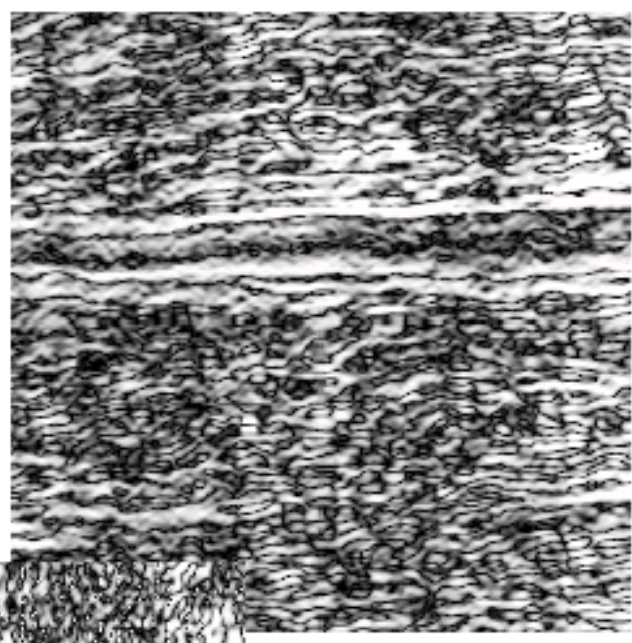

(b)

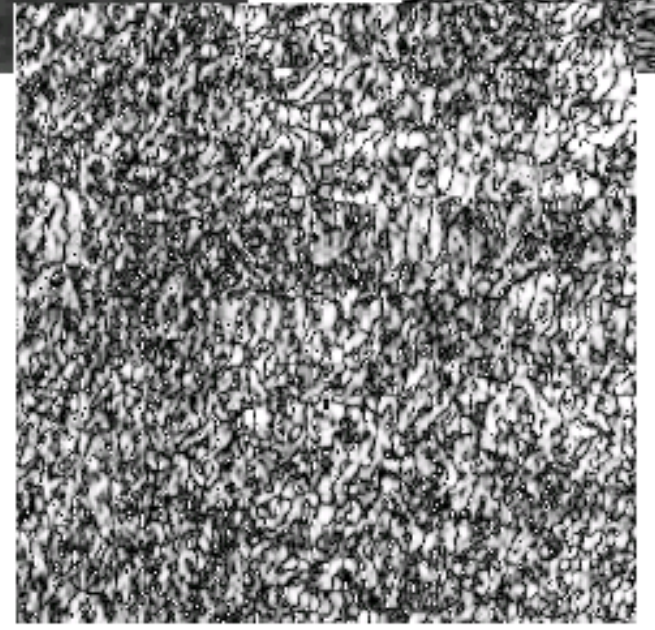

(c)

Figure 9. (a) Input slurry image with a streak. (b) $f_{\mathrm{d} 1}$ component of the MSWAR of the input image after2 iterations. (c) $f_{\mathrm{d} 2}$ component of the MSWAR after 2 iterations. Note that, due to the presence of the streak, the image appears more homogeneous in (c) than in (b) 


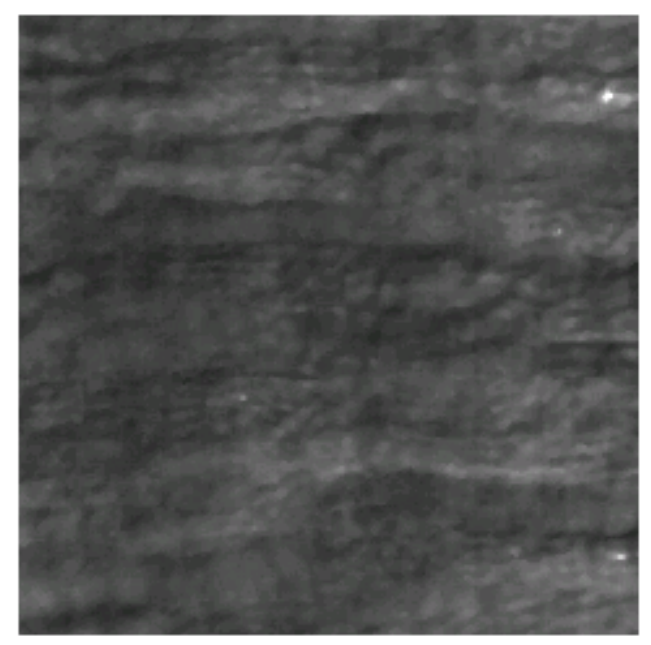

(a)

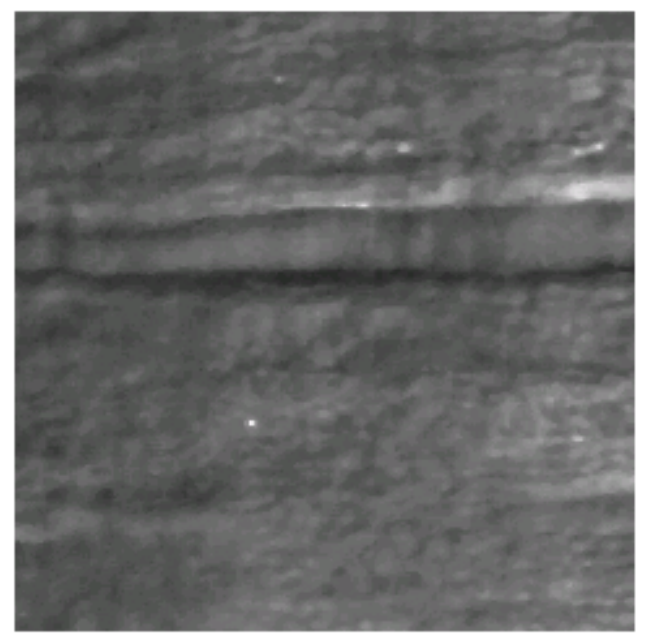

(c)

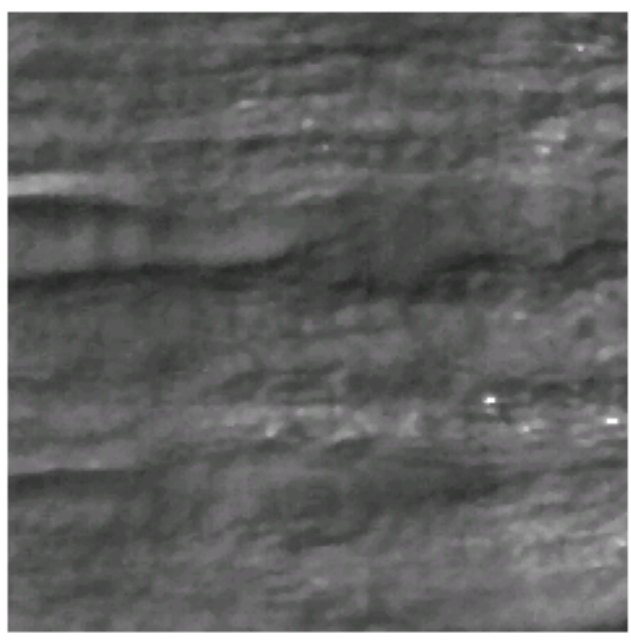

(b)

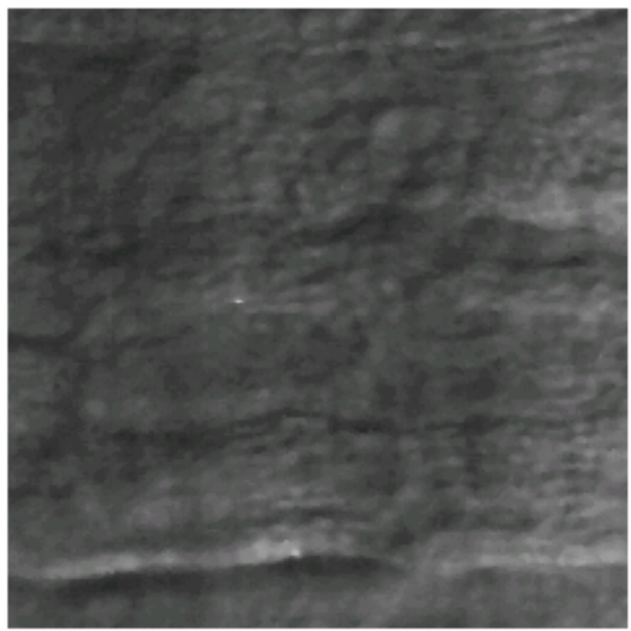

(d)

Figure 10. Four consecutive frames of slurry images showing the eventual formation of a streak in (b) and (c) and its disappearance in (d). Computed values of $V=663,5340,5803$, and 1285, respectively, indicate first a decrease in homogeneity values due to the appearance of the streak and then an increase due to its disappearance. 


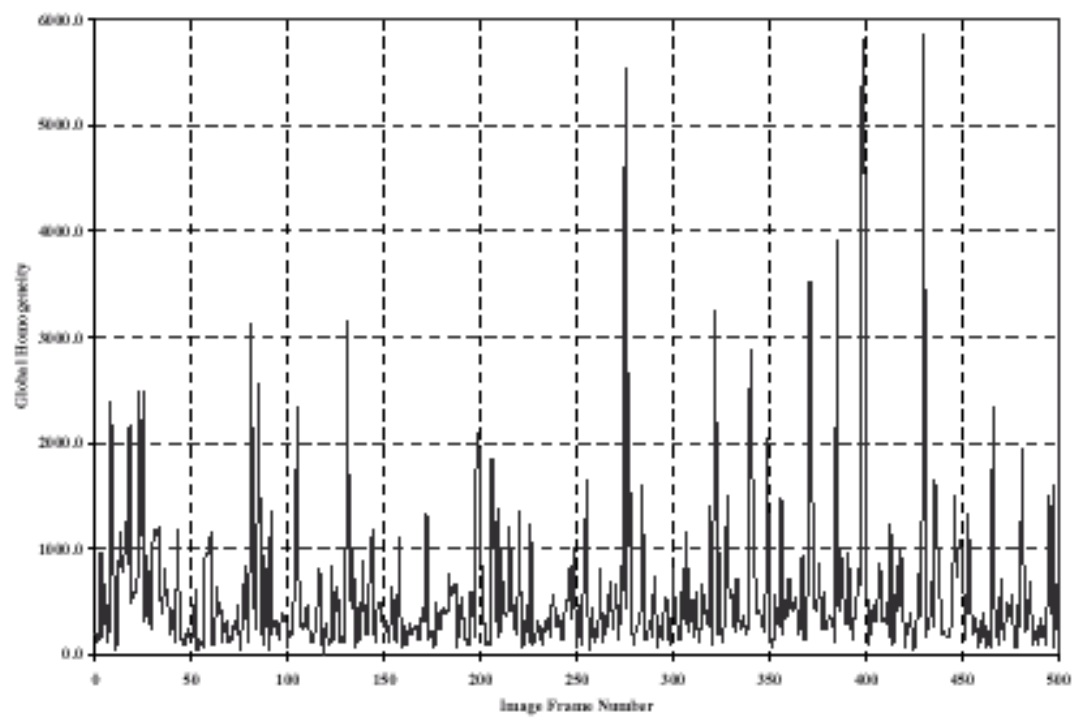

(a)

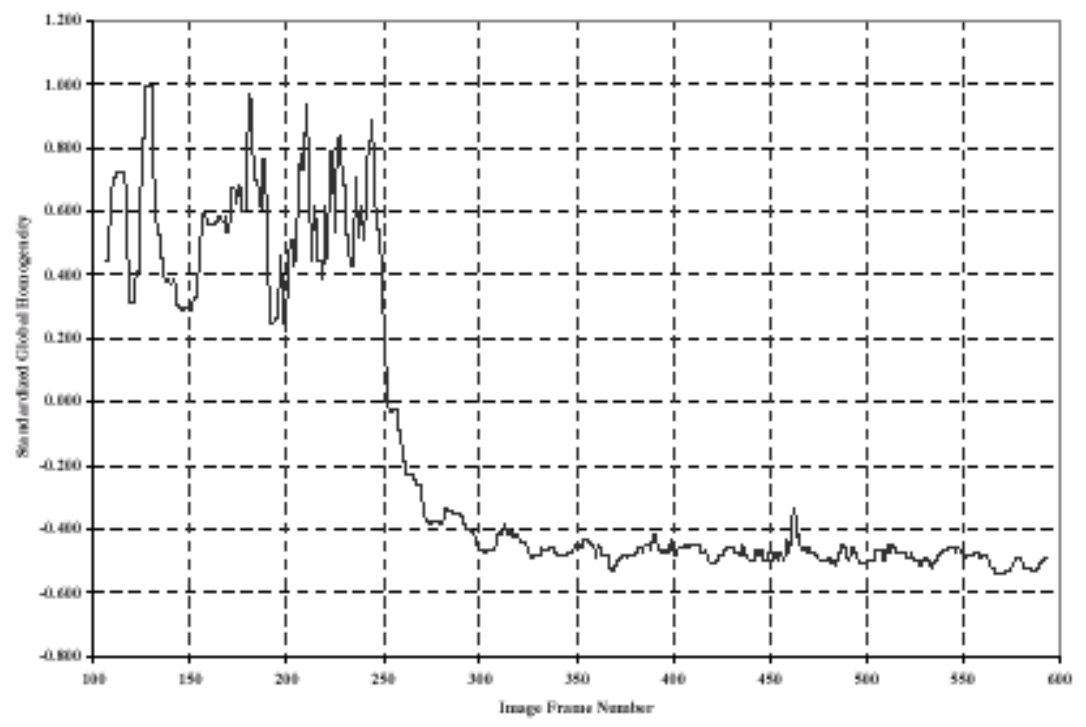

(b)

Figure 11. (a) Homogeneity measures for 500 consecutive images of slurry. Local maxima correspond to presence of streaks. (b) Moving average plot of standardized homogeneity measures for over 550 consecutive images. The sudden drop in values (i.e., increase in homogeneity) indicates an instance when the paper machine operator switched from slurry to water. 


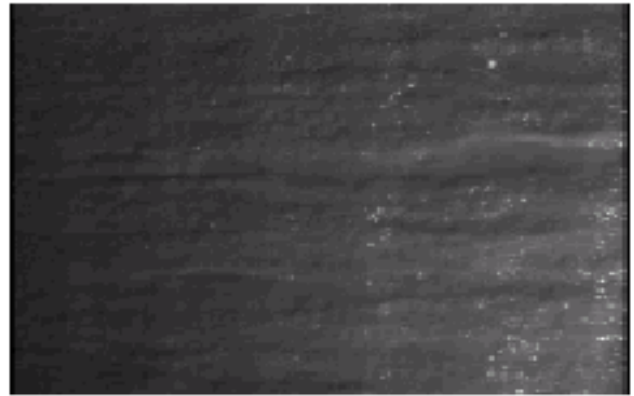

(a)

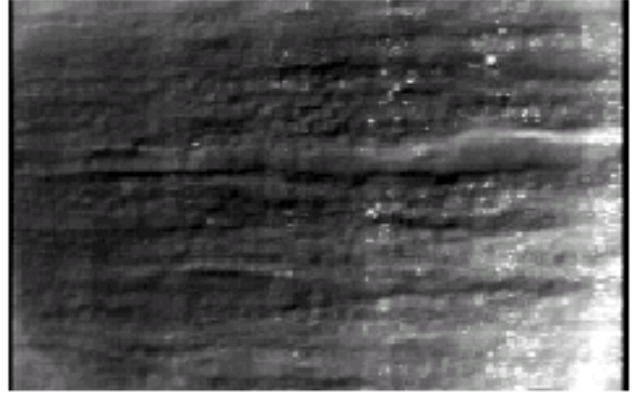

(b)

Figure 12. Intensity images of slurry (a) before and (b) after the application of the background subtraction algorithm.

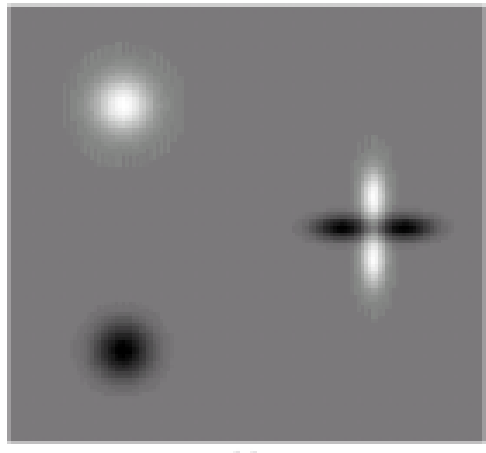

(a)

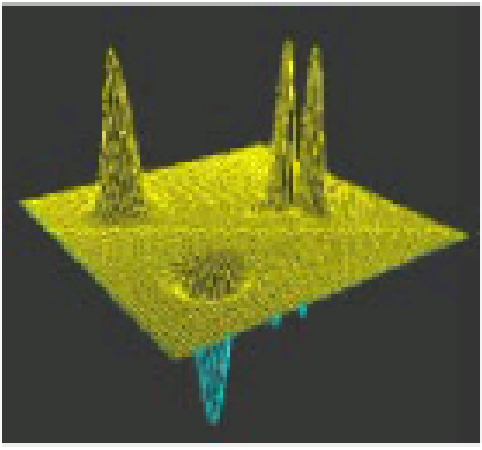

(b)

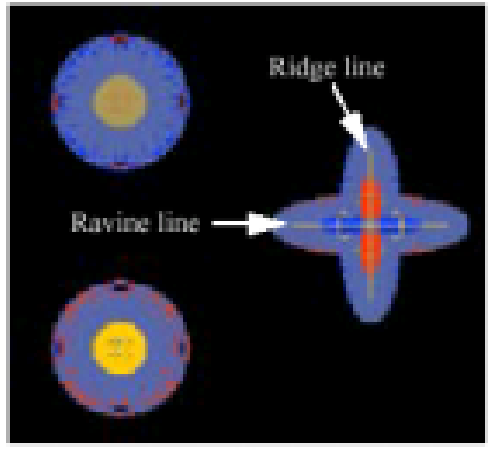

(c)

Figure 13. (a) Synthetic image. (b) 3-D representation of the intensity image in (a). (c) the labeled topographic image via the facet model. Each intensity value in (c) represents a different topographic structure, e.g., black represents flat regions. 


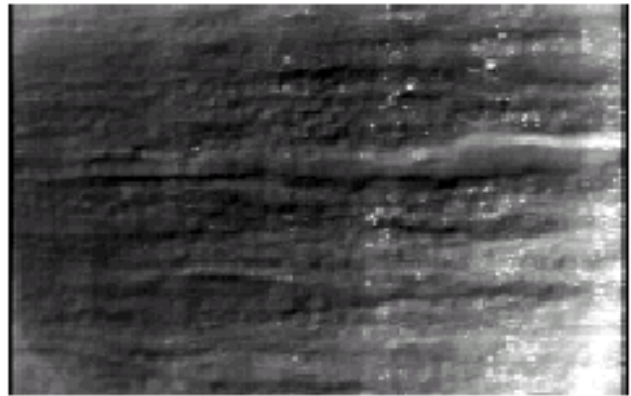

(a)

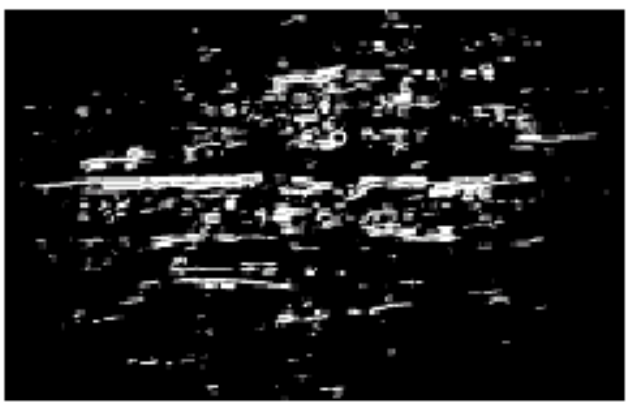

(c)

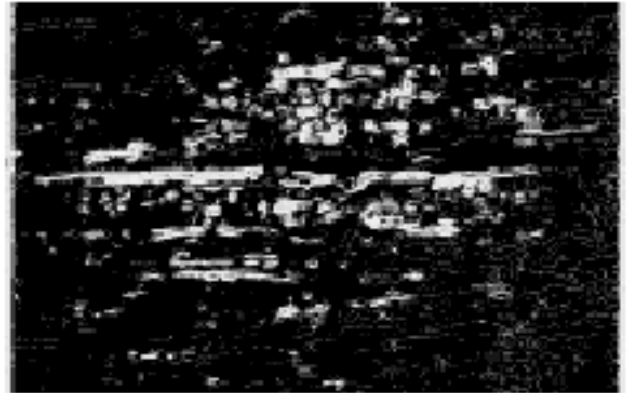

(b)

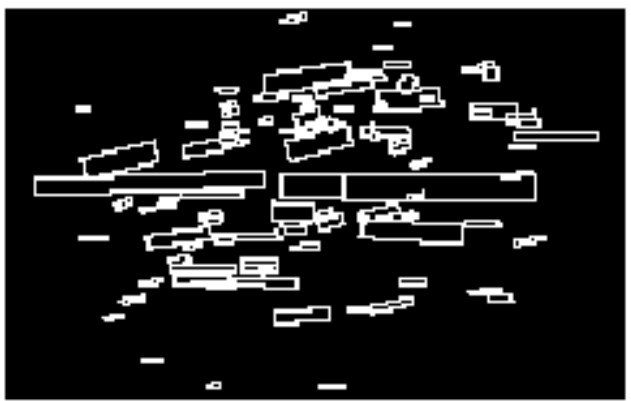

(d)

Figure 14. Output of the background subtraction algorithm. (b) Output of the facet generation step indicating pixels labeled as hillside convex, hillside concave, and hillside saddle structures. (c) Morphological linking of the horizontally-oriented structures and removing of noise. (b) Minimum bounding boxes enclosing the blobs in (c). The bounding boxes depicted here have been filtered for their sizes, orientation, and elongation. 

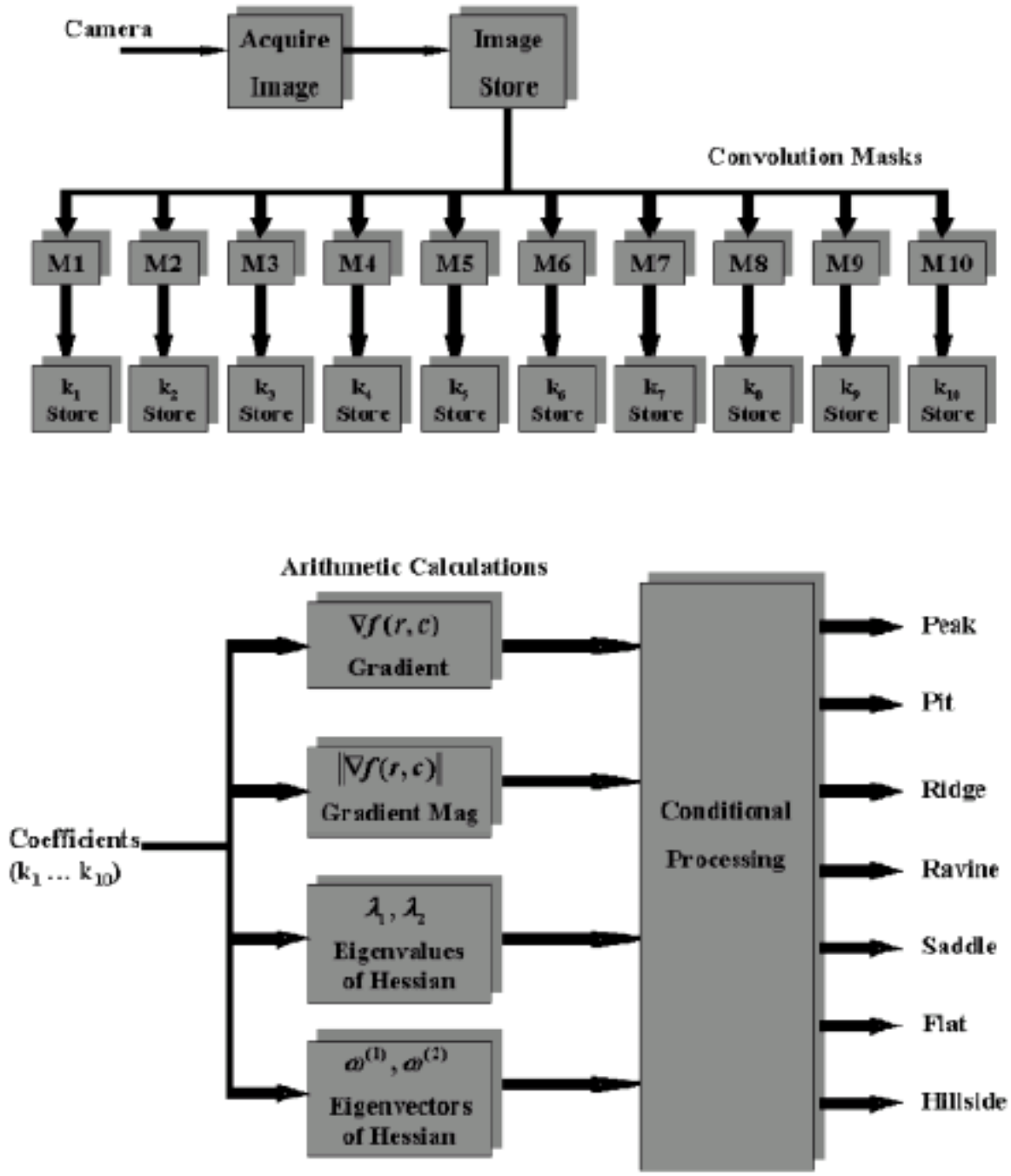

Figure 15. Block diagrams of the real-time implementation of the faced-based, streak localization algorithm. 